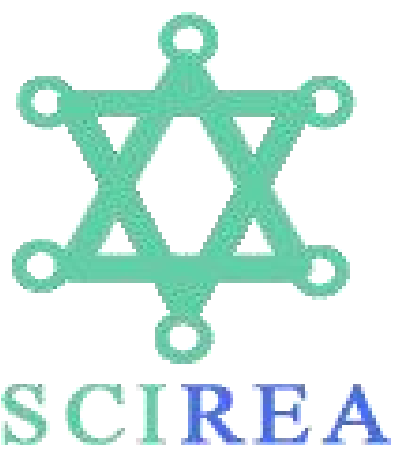

SCIREA Journal of Information Science

and Systems Science

http://www.scirea.org/journal/ISSS

October 25, 2021

Volume 5, Issue 5, October 2021

\title{
Quantum Qubit Information Conspiracy
}

\author{
Francis T. S. Yu \\ Emeritus Evan Pugh (University) Professor of Electrical Engineering, Penn State University, \\ University Park, PA 16802, USA \\ Email: fty1@psu.edu
}

\begin{abstract}
In this article I will show quantum qubit information transmission is as elusive as Schrodinger 's cat. Since there are two types of information transmission; namely Wiener and Shannon, I will start from mutual information to show that quantum qubit information-transmission is virtual as mathematics is, which is not existed within our temporal $(t>0)$ universe. Since quantum information transmission depends on Schrödinger's principle of superposition, which was derived based Schrödinger's equation. From which we see that Schrödinger's equation is a time independent formula only exists within an empty timeless $(t=0)$ space. From this I will show quantum qubit information is a noiseless channel which does not exist within our universe. Since quantum information is relied on Schrödinger's timeless fundamental principle, I shall show that qubit information is timeless $(\mathrm{t}=0)$ and fictious. In order to further illustrate what empty space paradigm has done to superposition, I will demonstrate what empty space can do to qubit information. Since physically realizable science depends on nature of our temporal $(t>0)$ universe, from which I will show where Schrödinger's quantum machine and his fundamental principle is situated. Since qubit information has emerged as a worldwide quantum conspiracy, it is hard to predict when this conspiracy will end. Since I am a scientist, I
\end{abstract}


wish this qubit conspiracy will be ended soon, since science is supposed to be physically realizable.

Keywords: Qubit information, Quantum information, Superposition principle, Quantum mechanics, Timeless space, Temporal space, Schrödinger equation, Quantum conspiracy.

\section{Quantum information-transmission}

Let us start with information transmission through an additive Gaussian noisy channel as depicted in Figure 1. In which we see that an input information $A=\left\{a_{i}\right\}$ is transmitting to the receiving end of $B=\left\{b_{j}\right\}$, where $i=1,2, \ldots M$, and $j=1,2, \ldots N$. Two key equivalent equations represent average amount of information transferred through the noisy Gaussian channel are given by [1],

$$
\begin{aligned}
& \mathrm{I}(\mathrm{A} ; \mathrm{B})=\mathrm{H}(\mathrm{A})-\mathrm{H}(\mathrm{A} / \mathrm{B}) \\
& \mathrm{I}(\mathrm{A} ; \mathrm{B})=\mathrm{H}(\mathrm{B})-\mathrm{H}(\mathrm{B} / \mathrm{A})
\end{aligned}
$$

where $\mathrm{I}(\mathrm{A} ; \mathrm{B})$ is the mutual information transmitted through the channel, $\mathrm{H}(\mathrm{A})$ represents the input information ensemble provided by the sender, H(A/B) is the information loss (or equivocation) through the channel due to noise, $\mathrm{H}(\mathrm{B})$ represents the information ensemble at the receiving end and $\mathrm{H}(\mathrm{B} / \mathrm{A})$ is noise entropy of the information channel.

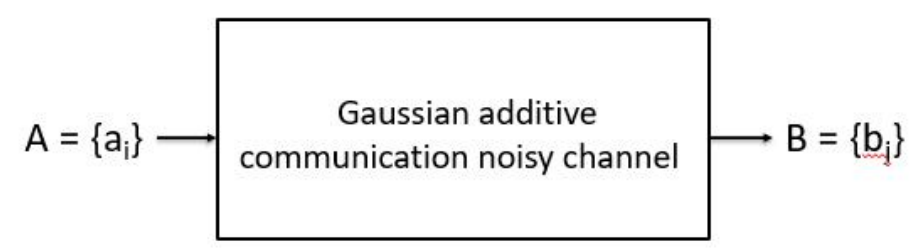

Figure 1 Shows an input-output Gaussian additive information channel. Where $A=\left\{a_{i}\right\}$ and $B$ $=\left\{b_{j}\right\}$ represent the input and output discrete-information ensembles.

However there is a distinction between these equations; one is used for "reliably" information transmission and the other is used to "retrieve or recover" the information that has been send. Nevertheless there are two types of disciplines or orientations, one is for information retrieval by Norbert Wiener [2,3] and the other is for reliable transmission by Claude Shannon [4]. In other words Wiener's communication strategy is that a sent information can be retrieved at the 
receiving end. Yet, Shannon's information-transmission carries a step further by encoding the information before it is transmitted, such that information can be "reliably" received at the receiving end. For example guessing what information that had been sent by the sender is Wiener communication strategy, and the other is to be protecting the information by the sender before it was sent is Shannon's orientation.

For example one of the easier ways to achieve Shannon type transmission is simply increasing the signal to noise ratio such that reliable information can be transmitted. And this is precisely what is currently digital communication is doing. Since digital information transmission is one of the lowest transmission rates for information transmission (i.e., I bit per transmission), the precise reason for using digital transmission must be that transmitted signal can be repeated or refreshed that analog or multi-level transmission cannot. For example, a CD can sequentially copy for hundreds of times, the last copy would be just as good as the first copy.

Nevertheless, within our temporal $(t>0)$ universe, everything has a price to pay, a section of time $\Delta \mathrm{t}$ and an amount of energy $\Delta \mathrm{E}$ (i.e., $\Delta \mathrm{E}, \Delta \mathrm{t}$ ) and it is not free. From which we see that one of the trivial prices to pay must be a section of time $\Delta t$, this means that it takes a longer time to transmit digital information than the same information in analog form. Although digital transmission has the lowest rate, but it is benefit of digital information that can be easily repeated. For which we see that by transmitting digital signal with light carrier has the overall advantage than analog transmission. It is trivial to see that one can trade a shorter section of time $\Delta \mathrm{t}$ for a wider $\Delta \mathrm{E}$ (or equivalently a broader bandwidth) or vice versa for a bit of digital information transmission. In which we see that $\Delta \mathrm{t}$ and $\Delta \mathrm{E}$ can be traded as given by $[5,1]$,

$$
\Delta \mathrm{t} \Delta \mathrm{E}=\mathrm{h}
$$

where $\mathrm{h}$ is the Planck's constant. Or equivalently we have,

$$
\Delta \mathrm{t} \Delta v=1
$$

where $\Delta v$ is the bandwidth of the carrier and $v$ is the frequency. In which we see that an unitinformation cell can be plotted as shown in Figure 2 . 


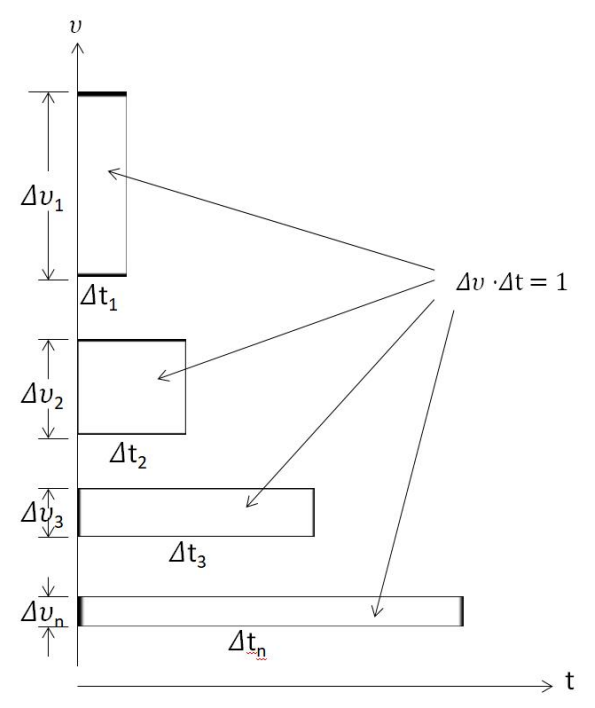

Figure 2 Shows the dynamics of $(\Delta v, \Delta \mathrm{t})$ Information cells; where $\Delta v_{\mathrm{n}}$ and $\Delta \mathrm{t}_{\mathrm{n}}$ are the bandwidths and timelimited sections, and $v_{1}>v_{2}>v_{3}>\ldots>v_{\mathrm{n}}$ are the carrier's frequencies. Where $\Delta v \Delta \mathrm{t}=1$ or equivalently $\Delta \mathrm{E}$ $\Delta \mathrm{t}=\mathrm{h}$

From which we see that each information cell (i.e., $\Delta v \Delta \mathrm{t}=1$ ) is equivalently equals to a quantum unit of information-transmission. And this quantum unit is lowest physical unit that cannot be lightly violated within our temporal $(t>0)$ universe, since every subspace takes a section of time $\Delta \mathrm{t}$ and an amount of energy $\Delta \mathrm{E}$ (i.e., $\Delta \mathrm{t}, \Delta \mathrm{E}$ ) to create and each unit of information cell. This means that $(\Delta \mathrm{t}, \Delta \mathrm{E})$ is limited by a quantum leap of light hv or a quanta that is,

$$
\Delta \mathrm{t} \Delta \mathrm{E} \geq \mathrm{h}
$$

Or equivalently,

$$
\Delta \mathrm{t} \Delta v \geq 1
$$

which is the well-known Heisenberg uncertainty principle [6]. Or equivalently equals to Yu's natural uncertainty principle [7] which means uncertainty changes naturally with time instead of limited by observation as Heisenberg assumed.

In view of the uncertainty relationship of Eq. (3) or Eq. (4), we see that every bit of information to be transmitted (or processing) is limited by a quantum unit of $(\Delta \mathrm{t}, \Delta \mathrm{E})$, which is the necessary cost that we have to pay which it is not free. However for a bit of information to be transmitted instantaneously (i.e., $\mathrm{t}=0$ ) it is required that $\Delta \mathrm{t}=0$. But the section of $\Delta \mathrm{t}$ no matter how small (i.e., $\Delta \mathrm{t} \rightarrow 0$ ) but never goes to zero (i.e., $\Delta \mathrm{t}=0$ ) is needed to transmit a bit of information. From which we see that if $\Delta \mathrm{t}=0$, then there has no signal presentation since it 
has no time to represent a signal which is timeless $(t=0)$. From which we see that it is impossible to transmit signal without a section of time $\Delta t$ within our temporal $(t>0)$ universe, since it takes a section of time $\Delta \mathrm{t}$ and an amount of energy $\Delta \mathrm{E}$ to transmit. And it is impossible to transmit a bit of information without the expenditure of a section of time $\Delta$ t no matter how small it is within our temporal $(\mathrm{t}>0)$ universe. Unless the transmission is within an empty timeless $(t=0)$ space but again how can a signal be represented without width. Since empty space cannot exist within our temporal $(\mathrm{t}>0)$ universe, it is trivial to see that instantaneous (i.e., $\mathrm{t}=0$ ) transmission hypothesis is not a physically realizable assumption within our universe. But it is possible to transmit signal without width (i.e., $\Delta t=0$ ) within an empty space, but $\Delta \mathrm{t}=0$ has no signal and no time.

From which we have shown that quantum qubit information-transmission is physically unrealizable even within an empty timeless $(t=0)$ space, although binary signal (e.g., 0 and $\mathrm{I}$ ) can be instantaneously and simultaneously transmitted within an empty space. But it is impossible to transmit signal instantaneously within our temporal $(t>0)$ universe, since every bit of information takes a section of time $\Delta \mathrm{t}$ no matter how small it is to transmit.

Since noise is always a natural thing that coexists with signal and in space, from which we note that noise is inherently coexisted with $\Delta \mathrm{E}$ and it is existed within any transmission channel within our universe. Either multiplicative or additive noise, they are constantly changing with time which can be approximated by $\Delta \mathrm{E}(\mathrm{t})+\mathrm{n}(\mathrm{t})$, where $\mathrm{n}(\mathrm{t})$ is assumed as an additive noise. From which we see that both $\Delta \mathrm{E}(\mathrm{t})$ and $\mathrm{n}(\mathrm{t})$ are temporal $(\mathrm{t}>0)$ substances change naturally with time, since everything within our universe is temporal.

\section{Qubit information channel}

With reference to mutual information of Eq. (1) and Eq. (2) as I see it, quantum information transmission is basically exploiting Wiener's information retrieval strategy for the purpose of qubit communication. Since at the receiving end receiver would anticipate a more ambiguous

digital signal (e.g., either 0 or 1 ) at the receiving end. Which has treated the receiving end entropy $\mathrm{H}(\mathrm{B})$ as the source of a transmitter entropy $\mathrm{H}(\mathrm{A})$ to determine the intended signal was provided. Since it was originated by the sender, by maximizing entropy H(B) under noiseless condition, the receiver can interpret the received signal (e.g., 0 or1) as equals to a qubit 
information. Which is the qubit principle that currently use for quantum communication and computing $[8,9]$.

Nevertheless it is not the qubit principle, but it is the physically realizable of the qubit information within our temporal $(\mathrm{t}>\mathrm{O})$ universe since everything has a cost to pay within our universe. Firstly quantum communication and quantum supremacy computing rely on Schrodinger's fundamental principle of superposition. In other words if the fundamental principle cannot exist within our universe, then there no sense to talk about the viable capability of qubit information can offer.

For which let us go back to the fundamental aspect of mutual communication principle of Eq. (1) and Eq. (2). Since the communication channel is assumed a binary (i.e., 0, 1) channel, then the information source $\mathrm{A}=\{0,1\}$ is a binary digital source. For which the source entropy $\mathrm{H}(\mathrm{A})=1$ bit per unit $\Delta \mathrm{t}$, no matter how small $\Delta \mathrm{t}$ is otherwise signal cannot be represented and transmitted. In which we see that this is one of the basic constraints of signal representation, otherwise it has no signal can be used regardless the paradigm is either timeless $(\mathrm{t}=0)$ or temporal $(\mathrm{t}>0)$.

Since quantum communication channel is situated within an empty space model as shown in the Figure 3, quantum information transmission is within in a timeless or no-time regime, then we see that binary source of $A=\{0,1\}$ is capable of transmitting 0 and 1 instantaneously and simultaneously within an empty space paradigm, since within empty space it has no time and no space. From which we see that the instantaneously and simultaneously transmission of 0 and 1 is precisely the Schrödinger's fundamental principle of superposition has stated. And this is exactly where qubit quantum information comes from an empty space paradigm of Figure 3.

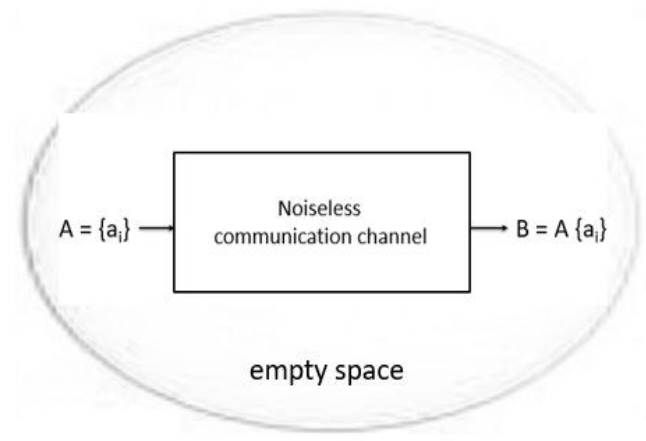

Figure 3 shows a conventional noisy communication channel is embedded within an empty space. But it is not a physically realizable paradigm since substance and emptiness cannot coexist. 
From which the set mutual information transmission equations can be reduces to,

$$
\begin{gathered}
I(A ; B)=H(A) \\
I(A ; B)=H(B)=H(A)
\end{gathered}
$$

Since quantum communication channel is embedded within an empty timeless $(t=0)$ space paradigm has no noise and no time, we have $\mathrm{H}(\mathrm{A} / \mathrm{B})=0$ and $\mathrm{H}(\mathrm{B})-\mathrm{H}(\mathrm{B} / \mathrm{A})=\mathrm{H}(\mathrm{A})$, because information loss due to noise $\mathrm{H}(\mathrm{A} / \mathrm{B})=0$ and $\mathrm{H}(\mathrm{B})>\mathrm{H}(\mathrm{A})$ has reduced to $\mathrm{H}(\mathrm{B})=$ $\mathrm{H}(\mathrm{A})$. From which we see that quantum qubit information in principle can be developed based on Wiener's information strategy under the noiseless consideration. Which has taken the output end H(B) entropy ensemble to interpret as the input H(A) ensemble for the intended sent signal (i.e., either 1 or 0 ). Which is equivalent to recovering the intended input signal that was corrupted by the noisy channel of Wiener's communication orientation.

However quantum information is dependent on Schrodinger's superposition principle such that binary transmission of 0 and 1 can transmit instantaneously and simultaneously, by which it is presented as a quantum bit or a qubit to determine the input source encoding of either 1 or 0 . Since the quantum information channel is assumed within an empty space paradigm, it is trivial to see that the operation is instantaneous and simultaneous but only within an empty space regime. In other words qubit information is a timeless $(t=0)$ communication strategy which only existed within an empty virtual space since it has no time and no space. Nevertheless qubit information is the anchor principle for quantum communication, but unfortunately qubit information not existed within our temporal $(t>0)$ universe.

There is a similar principle on quantum communication strategy is that a received signal dependent upon the binary scenario after observation as the paradox of Schrodinger's cat. In which the observer (i.e., the receiver) did not know the cat within the Schrödinger's box is either alive or dead until the observer opens the box. In which we see that it is the observer (i.e., the receiver) confirms the outcome after the observation. Although paradox of Schrödinger's cat that had have been debated for over 85 years since the disclosure of the Schrodinger's cat in 1935 and the paradox of superposition had had in doubt by Einstein and his collaborators and others [10], yet they had not found where the contradiction comes from until recently discovery of the temporal $(\mathrm{t}>0)$ universe $[11,12]$. From which I had shown that our universe is a time dependent space changes with time, but not change the time. Even though I had 
proven in preceding that qubit information is virtual, fictitious, and timeless $(t=0)$, but I will give a more convincing proof where the timeless $(t=0)$ superposition come from as fallows.

Since qubit information was developed from an empty space paradigm as shown in Figure 4, From which we see that it has represented a non-physically realizable paradigm to tackle with. As I had noted since the beginning of science, for centuries empty space paradigm had been inadvertently used for analyses and again similar mistake had been taken in Figure 4, a timeless $(t>0)$ subspace model. From which we see that within an empty space firstly it cannot a noisy channel. It is because it is a timeless $(t=0)$ channel, then the binary source of A can transmit 0 and 1 simultaneously and instantaneously since within an empty space it has no time and no space. Then it is a "perfect" communication channel why we bother to create qubit information for transmission, since information can be transmitted instantly without time limit and simultaneously superimposing the binary signal. But again, empty space has no time and no space, it is a virtual space not physically existed within our temporal $(t>0)$ universe. From which we see that qubit information is virtual as mathematics is, which cannot be implemented within our universe.

$$
\begin{aligned}
& \begin{array}{ll}
A=\{0,1\} \longrightarrow \begin{array}{ll}
\text { Timeless }(t=0) \text { quantum } \\
\text { communication channel }
\end{array} & \begin{array}{l}
B=\text { B }\{\text { qubit }\} \\
H(B)=1 \text { bit }
\end{array} \\
&
\end{array} \\
& \text { Empty space }
\end{aligned}
$$

Figure 4 Shows a binary timeless $(t=0)$ quantum qubit-information channel. Since it is timeless, the channel is noise free. $A=\{0,1\}$ represents an input binary source, $H(A)=1$ bit is the input entropy, $B=B$ qqubit $\}$ is output quantum bit, $H(B)=$ qubit is the output entropy in qubit. Since quantum qubit information transmission has treated the input binary source $\mathrm{A}=$ $\{0,1\}$ as a qubit output $B=\{$ qubit $\}$, at the receiving ending information can be presented in quantum bit or qubit.

Nevertheless, if the qubit information transmission channel is situated within a temporal $(\mathrm{t}>0)$ subspace as shown in Figure 5, then the responds of qubit information is subjected to the boundary condition within temporal $(\mathrm{t}>0)$ subspace. In which we see that simultaneous and instantaneous superposition of binary digital transmission (i.e., 0,1 ) fails to exist. From which we see that entropy $\mathrm{H}(\mathrm{B})$ at the transmitted end cannot be treated as qubit information since 
superposition does not exist within our temporal $(t>0)$ universe. Then output end ensemble is $\mathrm{B}=\{0,1\}$ which is the same as a conventional noisy binary channel.

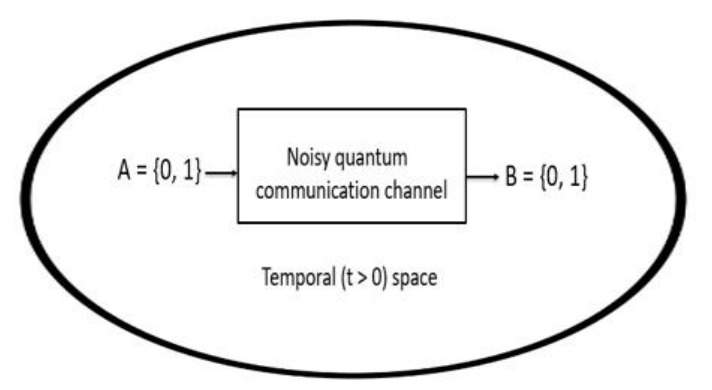

Figure 5 Shows a binary noisy quantum communication channel embedded within a temporal $(t>0)$ space. For which output entropy is always larger than the input entropy that is $H(B)>$ $\mathrm{H}(\mathrm{A})$.

\section{Schrödinger equation to Nonexistent Superposition}

Since Schrödinger's quantum mechanics is the legacy of Hamiltonian [13], by adopting Bohr's quantum leap state energy $\mathrm{E}=\mathrm{h} v$ from Bohr's atomic model [14], Schrödinger's equation is given by,

$$
\frac{\partial^{2} \Psi}{\partial x^{2}}+\frac{8 \pi^{2} \mathrm{~m}}{h^{2}}(E-V) \Psi=0
$$

where $\psi$ is the wave function has to be determined, $\mathrm{m}$ is the mass of a photonic-particle (i.e., photon), $\mathrm{E}$ and $\mathrm{V}$ are the dynamic quantum state energy and potential energy of the particle, $\mathrm{x}$ is the spatial variable and $\mathrm{h}$ is the Planck's constant.

From which we see that Schrödinger equation is a point-singularity deterministic timeindependent, timeless $(t=0)$, or no-time equation. Which is virtually identical to Hamiltonian equation, except the energy factor; $\mathrm{E}=\mathrm{h} v$ quantum state energy of Bohr as shown in Figure 6. This is precisely the reason why Schrödinger's theory is known as quantum mechanics. And the names sake of mechanics must be the legacy of Hamiltonian. 


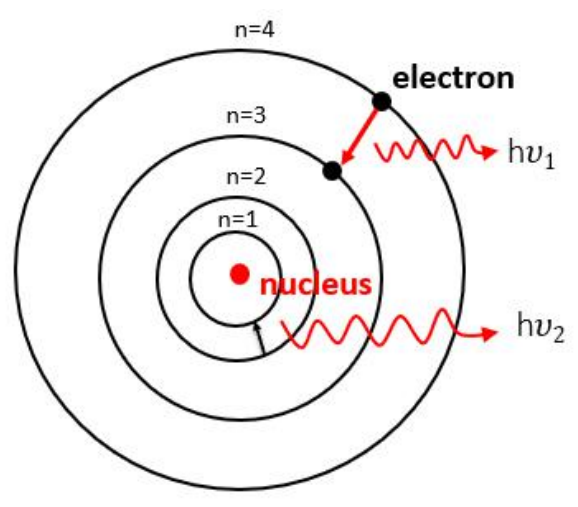

Empty space

Figure 6 Shows a typical multi-quantum state leaps Bohr's atomic model embedded within an empty space. $\mathrm{h} v_{\mathrm{n}}$ is quantum leap, $\mathrm{n}=1,2$.

Beside Schrödinger equation is a point-singularity approximated, since without singularity presentation it would be very difficult to present in rigorous mathematical form. And this is precisely all scientific equations are in point-singularity approximated. But it is a deterministic time-independent, timeless $(t=0)$, or no-time equation that causes the physically realizability of Schrödinger equation. Nevertheless time independent or timelessness laws were coming from an inadvertently empty subspace paradigm; that we had have using it for centuries. Which was from the background subspace of a piece paper that we had have commonly used.

Since fundamental principle of superposition is the core of Schrödinger's quantum mechanics, without the core it will be very difficult to justify the legacy of his principle. Aside the legacy from timeless $(\mathrm{t}=0)$ Hamiltonian, it is Bohr's atomic model as presented within an empty space that caught my attention, how can quantum leaps radiate since the atomic model is embedded within an empty space? Nevertheless, as from physically realizable standpoint, all the quantum leaps $\mathrm{h} \sqrt{n}_{\mathrm{n}}$ are supposed to be time and band limited. From which we see that Schrödinger's equation only existed within an empty timeless $(t=0)$ space that includes his superposition principle.

\section{What empty space does to quantum wavelets.}

Although timeless $(t=0)$ space is not a physical realizable paradigm, but quantum physicists can implant particles into an empty space since theoretical physicists are also mathematicians. And this is precisely that quantum theory behaves strangely that Einstein and his colleagues had opposed severely [10]. Yet quantum principle persisted over decades and currently has 
having the possibility to take over the supremacy of digital computing. Nevertheless, given two isolated particles embedded within an empty space shown in Figure 7(a), we see that these particles superimposing at time $\mathrm{t}=0$, since within an empty space it has no time. Because empty space has no time it has no space, we see that particle 1 and 2 are physically superposing together all over the entire unbounded empty space as illustrated in Figure 7(b). From which we see that this is exactly what superposition of Schrödinger does for particles but only within an empty virtual space.

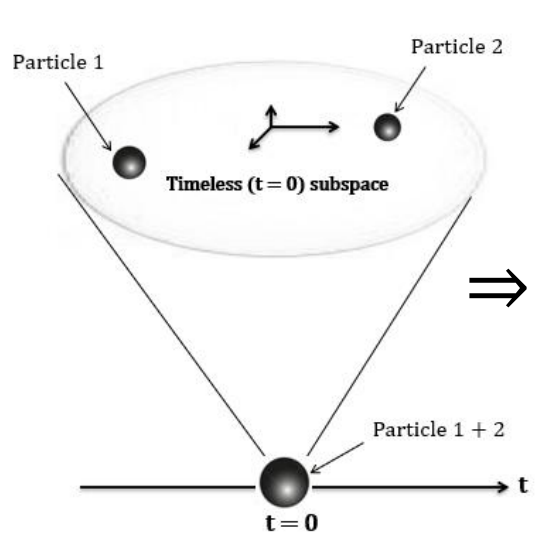

(a)

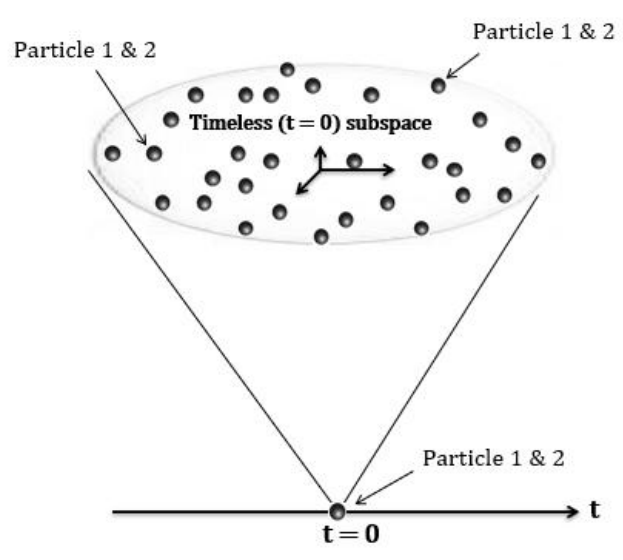

(b)

Figure 7 Shows two isolated particles are embedded within an empty. (a) shows particle 1 and 2 corrupted at $\mathrm{t}=0$. (b) shows particle 1 and 2 are superimposing together over the entire empty space and corrupted at $\mathrm{t}=0$.

Since quantum leap energy $\mathrm{E}=\mathrm{h} v$ is not a physically realizable hypothesis, we see that multiquantum leaps $\mathrm{h} v_{\mathrm{n}}$ will be superimposed at all time, regardless of it is within a virtual timeless $(t=0)$ or a temporal $(t>0)$ subspace. But from physical reality, every quantum leap energy has to be time and band limited (i.e., $\Delta \mathrm{E}=\mathrm{h} \cdot \Delta v$ ) within our temporal $(\mathrm{t}>0)$ space, for which we see that for time-limited equations it is very unlikely that the quantum wavelets will be superimposed together at the same time.

For example, we assume a set of time-unlimited quantum state wave functions as given by,

$$
\begin{aligned}
& \psi_{1}(\mathrm{t})=\psi_{01} \exp \left[-\mathrm{i} 2 \pi v_{1}\left(\mathrm{t}-\mathrm{t}_{01}\right) / \mathrm{h}\right] \\
& \psi_{2}(\mathrm{t})=\psi_{02} \exp \left[-\mathrm{i} 2 \pi v_{2}\left(\mathrm{t}-\mathrm{t}_{02}\right) / \mathrm{h}\right]
\end{aligned}
$$

where $\psi_{0 \mathrm{n}}$ and $\mathrm{t}_{0 \mathrm{n}}$ are arbitrary constant and time constant respectively, $v_{\mathrm{n}}$ is the frequency, and $\mathrm{h}$ is the Planck's constant. It is trivial to see that this set wave functions are not temporal 
function that cannot be implemented within our temporal $(t>0)$ universe. Yet it is possible to reconfigure them to become time-limited Gaussian wavelets as given by,

$$
\begin{aligned}
& \psi_{1}(t)=\psi_{01} \exp \left[-\alpha_{01}\left(t-t_{01}\right)^{2}\right] \cos \left(2 \pi v_{1} t\right), t>0 \\
& \psi_{2}(t)=\psi_{02} \exp \left[-\alpha_{02}\left(t-t_{02}\right)^{2}\right] \cos \left(2 \pi v_{2} t\right), t>0
\end{aligned}
$$

where $\alpha_{\text {on }}$ is an arbitrary constant. Since this set equations are temporal $(\mathrm{t}>0)$ wavelets, they can be implemented within our temporal $(\mathrm{t}>0)$ universe as shown in Figure 8 . In which it shows that wavelets are not superimposing together within temporal $(t>0)$ space. As in contrast within an empty space scenario shown in Figure 9, In which we see that superposition fails to exist within our temporal $(t>0)$ universe.

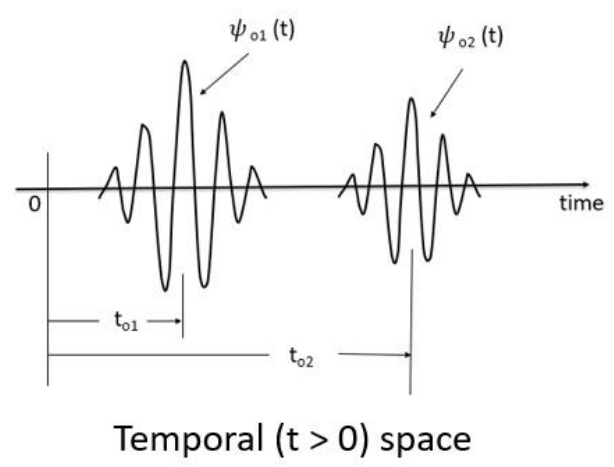

Figure 8 shows two distinctive quantum wavelets are separately existed at different time within a temporal $(t>0)$ space.

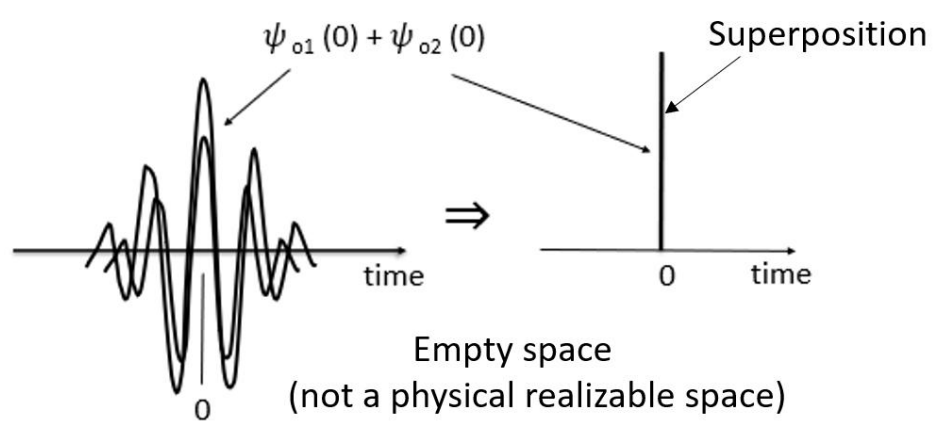

Figure 9 Shows what an empty space can do to quantum wavelets. First shows two quantum wavelets superimposing at $\mathrm{t}=0$ on the left-hand side diagram and then corrupting at time $\mathrm{t}=0$ as depicted on the right-hand side. For which we see that; superposition exists only within an empty timeless $(t=0)$ space.

\section{Nature of temporal $(t>0)$ universe}

It is essential to know the time-space we are living in, otherwise the predicted science may not physically realizable. For which Figure 10 is to epitomize the nature of our temporal $(t>0)$ 
universe, in which we see that our universe started from a big bang creation is about 14 billion light years (BLY) old. In which we see that Past time $(t<0)$ is a certainty domain that includes all past virtual universes but without physical substance and time in it. Future time domain $(t>0)$ represents a physical realizable uncertainty time-space, where space and time are coexisting. In other words our universe is constantly changing with time and cannot stop the changes. nevertheless our universe cannot curve the time-space as Einstein's general theory tells us it could [15]]]]]]. While $\mathrm{t}=0$ represents an instant moment of absolute physical certainty of our universe, which this is the only moment that everything within our universe is absolute certainty. But this present moment $\mathrm{t}=0$ will quickly moves on be came next instant moment (e.g., $\mathrm{t}=0+\Delta \mathrm{t}$, where $\Delta \mathrm{t} \rightarrow 0$ ) of absolute certainty.

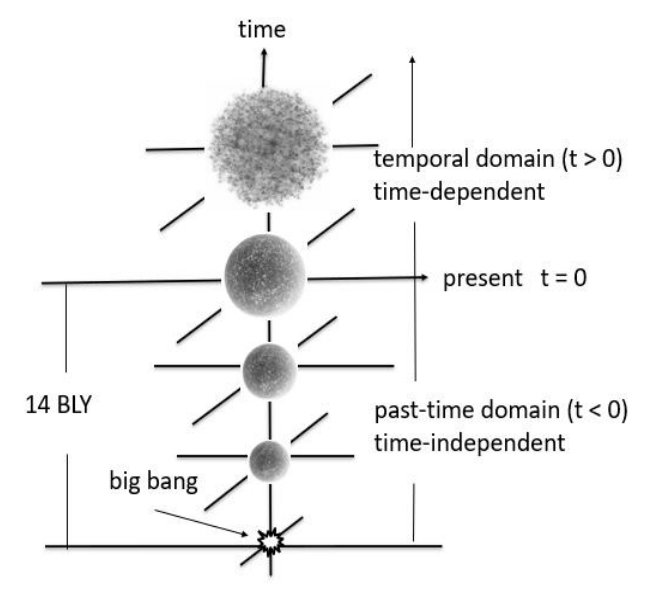

Figure 10 Shows our universe started from big bang creation is about 14 billion light years (BLY) old. Past time domain $(t<0)$ represents some certainty virtual universe which has no physical substance and time. Future time domain $(t>0)$ represents a physical realizable uncertainty universe, and $t=0$ represents an instant moment of absolute physical reality of our universe.

Since past certainties had had changed at specific past-times but do not have any physical substances and no time with them, we see that the past certainties are independent with time, but time is no longer existed within the past $(t<0)$ domain. And this is precisely where our laws, principles, and theories were developed. For example, use past certainty to predict deterministic future, but future is supposed to be indeterministic or uncertainty.

Nevertheless, within the future time domain [i.e., temporal $(\mathrm{t}>0)$ universe] it is our universe changes with time instead of universe changes (i.e., curves) the space-time. In which we see that future time domain (i.e., temporal universe) is not deterministic. Therefore it is a serious mistake to treat our temporal $(\mathrm{t}>0)$ universe as a four-dimensional space-time continuum as most scientists do. 
Yet it is this present moment $(t=0)$ divides the virtual past-time certainty domain $(t<0)$ and future uncertainty temporal $(t>0)$ domain. In other words all the past time universes are virtual images (i.e., information) with no physical substance and no time. While future time domain or temporal $(\mathrm{t}>0)$ universe is a physically realizable domain, for which we see that our universe physically changes with time. In view of this instant moment of $t=0$, we found that this is precisely Schrödinger's fundamental principle situated at $t=0$, where clock stop. From which we see that qubit quantum information can be transmitted freely without the expense of a section of time (i.e., $\Delta t=0$ ) at time $t=0$. But, within our universe there is always a price to pay, a section of time $\Delta \mathrm{t}$ and an amount of energy $\Delta \mathrm{E}$. Nevertheless it is the $\Delta \mathrm{t}$ that we cannot squeeze to zero (i.e., $\Delta \mathrm{t}=0$ ) even at the expense of huge amount of energy $\Delta \mathrm{E}$.

\section{Conclusion}

In conclusion I have shown that qubit information is as virtual as Schrodinger 's timeless $(\mathrm{t}=$ 0 ) fundamental principle of superposition which is only existed within an empty space and it is not existed within our temporal universe. Since quantum qubit information depends on Schrödinger's principle of superposition, which was derived based Schrödinger's equation. But Schrödinger equation is a legacy of Hamiltonian, we see that Schrödinger's equation is time independent. From this I have shown qubit information is a noiseless channel does not exist within our universe. One of the most important aspects within our temporal $(t>0)$ universe is that time cannot be change or even stop. From which I have shown that Schrodinger's quantum machine is timeless $(\mathrm{t}=0)$ as well as qubit information. Since there are two types of information transmission; namely Wiener and Shannon, I have shown qubit transmission is mostly Weiner information retrieval type, it requires more post processing that further deviates away from instant transmission merit. Nevertheless it must be the non-physically realizable quantum qubit information that has emerged as a worldwide quantum conspiracy, for which it is hard to predict when this conspiracy would be ended, since future prediction is not deterministic. Yet I am hoping that this fictious qubit information supremacy would be resolved since science is supposed to be physically realizable. 


\section{References}

[1] F. T. S. Yu, Optics and Information Theory, Wiley -Interscience, New York, 1976.

[2] N. Wiener, Cybernetics, MIT Press, Cambridge, MA, 1948.

[3] N. Wiener, Extrapolation, Interpolation, and Smoothing of Stationary Time Series, MIT Press, Cambridge, MA, 1949.

[4] C. E. Shannon and W. Weaver, The Mathematical Theory of Communication, University of Illinois Press, Urbana, IL, 1949.

[5] D. Gabor, "Communication Theory and Physics," Phil. Mag, vol. 41, no. 7, 1161, 1950.

[6] W. Heisenberg, "Über den anschaulichen Inhalt der quantentheoretischen Kinematik und Mechanik," Zeitschrift Für Physik, vol. 43, no. 3-4, 172 (1927)

[7] F. T. S. Yu, "Nature of Temporal ( $t>0$ ) Quantum Theory: Part II" Quantum Mechanics, Edited by P. Bracken, Chapter 9, p 161-188, IntechOpen, London (2020).

[8] C. H. Bennett, Quantum information and computation. Phys. Today, Vol.48, No.10, 24-30, 1995.

[9] K. Życzkowski,, P. Horodecki, M. Horodecki, and R. Horodecki, "Dynamics of quantum entanglement". Phys. Rev. A 65, 1-10, 2001.

[10] A. Einstein, B. Podolsky, and N. Rosen, "Can Quantum-Mechanics Description of Physical Reality Be Considered Complete?” Phys. Rev. Vol. 47, No. 10, 777-780, 1935.

[11] F.T.S. Yu, “Time: The Enigma of Space”, Asian Journal of Physics, Vol. 26, No.3, 143-158, 2017.

[12] F.T.S. Yu, "From Relativity to Discovery of Temporal ( $\mathrm{t}>0$ ) Universe", Origin of Temporal $(t>0)$ Universe: Correcting with Relativity, Entropy, Communication and Quantum Mechanics, Chapter 1, CRC Press, New York, 1 -26(2019).

[13] D. F. Lawden, The Mathematical Principles of Quantum mechanics, Methuen \& Co Ltd., London (1967).

[14] N. Bohr, "On the Constitution of Atoms and Molecules", Philos. Mag., Vol.26, No. 1, 1-23, 1913.

[15] A. Einstein, Relativity, the Special and General Theory, Crown Publishers, New York, 1961. 